LETTER

\title{
Respiratory therapists in a primary role as disease manager
}

\author{
Wendy Fascia MA, RRT ${ }^{1}$, Jennifer Pedley BS, RRT $^{2}$
}

W Fascia, J Pedley. Respiratory therapists in a primary role as disease manager. Can J Respir Ther 2018;54(4):75-76. doi: 10.29390/ cjrt-2018-023.

\begin{abstract}
At Crouse Hospital in Syracuse, New York, the Lung Partners (LP) program is being utilized to positively impact the management of a health population (chronic obstructive pulmonary disease, COPD) within our community. LP is a comprehensive, Primary Respiratory Care model for inpatient COPD disease management, currently comprised of 27 Respiratory Therapists (RTs) who are disease management specialists, having completed an extensive training program. There are over 600 COPD patients enrolled in our program, and in our database, which is approximately $25 \%$ of the diagnosed COPD population in our community.
\end{abstract}

The LP program is taking a different approach to improve the management of COPD both in and out of the hospital setting. In our program, RTs are positioned as the major physician partners in the management of COPD for all patients. Most individuals diagnosed with COPD are often very complex health care patients who see a variety of providers and social services on an outpatient basis, and then once in our hospital system they may see several different hospitalists during an inpatient hospital admission and also on each readmission. The numerous providers caring for these individuals on an occasional and brief basis, frequently results in delays in care due to communication issues. The many entities involved may also interfere with a clear plan at discharge, resulting in that plan not being carried out at home. Care becomes very fragmented. With our LP program, the goal is to decrease this fragmentation of care for these clients.

The LP program begins with the voluntary enrollment of inpatient clients who have a diagnosis of COPD and meet criteria for our LP program. They are then immediately partnered with a Primary LP RT, and also a Secondary RT for times when the Primary RT is not in-house. That Primary RT will have a primary relationship with that client with COPD for all hospital admissions, and then is actively involved in the client's transition to home. These LP RTs, knowing their client on an ongoing basis, have an insight into their routine maintenance, their triggers, and the barriers they face each day outside of the hospital, which is all vital information in developing a plan for discharge that will be effective in keeping that client from returning to the hospital for an unnecessary readmission.

To accomplish our program goals, (as spelled out in our Lung Partner Handbook), partnering the LP RT with the client is only the first step towards creating the connection necessary for this program to be successful. The Primary RT educates the inpatient client on their disease and coaches disease management skills. They screen them for co-morbidities that are, at times, poorly addressed in the hospital setting. Anxiety and depression are major co-morbidities, especially for this health population, and are part of the screening assessment by the Primary RT. The LP program collaborates with a psychology group to address this issue with identified clients. Other screenings in the initial assessment include nutrition, health literacy, and quality of life, among others that lead to conversation, producing opportunities to dig into the barriers that the client faces to maintaining their health at home. Protocols are in place for clients to receive services based on the Primary RT's assessment so care can be easily implemented and adjusted to fit the specific inpatient client's current condition. The continual day-to-day presence and reinforcement by the Primary/Secondary RT, moves the partnership between RT and client towards more of a bond/trust. This is all vital to the success of these individuals maintaining compliance with the discharge plan and remaining healthy at home.

The LP Team is notified the moment these individuals enter our emergency department (ED) or elsewhere in our hospital, as a patient. We have an alert built into our EMR that flags the LP Team immediately. This helps to maintain that trust and confidence in our "partnership" with these clients when a familiar person (their Primary/Secondary RT) shows up in the ED or their room soon after their arrival to the hospital. Their stay in the ED does not always lead to an admission if we can decipher the barrier that brought them there. Knowing these individuals well, along with their barriers to staying home, many times we can remedy the issue at that point in time and then avoid an unnecessary readmission. The ED physicians utilize the LP RTs and our knowledge of these patients and rely on them to intervene as they have been trained.

Through our Quality Improvement (QI) Department we measure the 30-day readmission rates for respiratory diseases on LP patients, which are those individuals we can directly influence. Hospital-wide CMS 30-day all-cause COPD readmissions are also evaluated. With our program in place we have shown a decrease in 30-day COPD readmission rates. For LP patients we saw a significant reduction in 30-day readmissions due to respiratory diseases by $28 \%(p=0.0176)$. We also saw a significant reduction in total COPD CMS readmissions by $24 \%$ $(p=0.045)$. We presented our data at the annual meeting of the American Association for Respiratory Care's Open Forum in October 2016 [1]. Our QI department also measures and reports on many other variables important to the success of this program, such as length of stay (LOS). For our LP patients we have seen a decrease in length of stay from 6.2 days to 5.8 days.

Currently the main LP program is an inpatient program in our hospital. We continually strive to expand our reach into the community to increase our ability to impact the management of this health population. New concentrations for our program include testing of our LP COPD patients for Alpha One Antitrypsin Deficiency, collaborating with many provider groups in the community on adherence and understanding of National COPD Guidelines, and addressing our high utilizers of health care with a multidisciplinary team. LP RTs

\footnotetext{
${ }^{1}$ Manager Respiratory, EEG, ECMO Programs, Crouse Hospital, 736 Irving Ave, Syracuse, NY

${ }^{2}$ Supervisor Respiratory, EEG/ Crouse Hospital, 736 Irving Ave, Syracuse, NY 13210/315-470-7133 jenniferpedley@crouse.org

Correspondence: Wendy Fascia MA, RRT, RT-NPS, Manager Respiratory, EEG, ECMO Programs, Crouse Hospital, 736 Irving Ave, Syracuse, NY 13210

Tel: 315-470-2689 Email:wendyfascia@crouse.org
}

This open-access article is distributed under the terms of the Creative Commons Attribution Non-Commercial License (CC BY-NC) (http:// creativecommons.org/licenses/by-nc/4.0/), which permits reuse, distribution and reproduction of the article, provided that the original work is properly cited and the reuse is restricted to noncommercial purposes. For commercial reuse, contact editor@csrt.com 
Fascia and Pedley

are also placed in an offsite capitated program that has contracted with our hospital to utilize the disease management skills of these RTs to help manage the care and control the high costs of this patient population.

By placing our RTs in a Primary Respiratory Care model we were able to reduce 30-day readmission rates, and decrease LOS for this patient population. The RT department has moved from a task oriented to disease management focus and utilizes RTs to the full extent of their licensure. RTs can be very successful in this role.

\section{REFERENCE}

1. Acevedo R, Fascia W, Raut L, Pedley J. Lung partners impact on reduction in 30-Day COPD readmissions rates. Respir Care. 2016 Oct; 61(10):OF33-OF33. 